\title{
Simultaneous measurement of patterns in the signal and idler near and far fields from a confocal optical parametrical oscillator with applications in quantum optics
}

\author{
Lassen, Mikael Østergaard; Tidemand-Lichtenberg, Peter; Buchhave, Preben
}

Published in:

Physical Review A

Link to article, DOI:

10.1103/PhysRevA.72.023817

Publication date:

2005

Document Version

Publisher's PDF, also known as Version of record

Link back to DTU Orbit

Citation (APA):

Lassen, M. Ø., Tidemand-Lichtenberg, P., \& Buchhave, P. (2005). Simultaneous measurement of patterns in the signal and idler near and far fields from a confocal optical parametrical oscillator with applications in quantum optics. Physical Review A, 72(2), 023817. https://doi.org/10.1103/PhysRevA.72.023817

\section{General rights}

Copyright and moral rights for the publications made accessible in the public portal are retained by the authors and/or other copyright owners and it is a condition of accessing publications that users recognise and abide by the legal requirements associated with these rights.

- Users may download and print one copy of any publication from the public portal for the purpose of private study or research.

- You may not further distribute the material or use it for any profit-making activity or commercial gain

- You may freely distribute the URL identifying the publication in the public portal 


\title{
Simultaneous measurement of patterns in the signal and idler near and far fields from a confocal optical parametrical oscillator with applications in quantum optics
}

\author{
Mikael Lassen, ${ }^{*}$ Peter Tidemand-Lichtenberg, and Preben Buchhave \\ Department of Physics, Technical University of Denmark, DK-2800 Lyngby, Denmark \\ (Received 4 February 2005; revised manuscript received 12 May 2005; published 18 August 2005)
}

\begin{abstract}
We present the results of an experimental investigation of multimode intensity patterns from an optical parametric oscillator operating above threshold and show that it oscillates in 10-15 transverse modes strongly coupled through the nonlinear crystal, which makes this setup useful for future investigation of quantum correlations in the transverse plane. We describe the experimental setup for simultaneous measurements of signal and idler near- and far-field patterns and analyze the effects of various experimental complications such as walk-off and thermal index changes on the generated patterns. We also show that the oscillator can be stabilized by optical feedback, indicating a possible route for controlling the generated intensity patterns.
\end{abstract}

DOI: 10.1103/PhysRevA.72.023817

PACS number(s): 42.60.Jf, 42.65.Yj

\section{INTRODUCTION}

Self-organized patterns created by nonlinear effects occur in many physical systems and have long been the subject of intense study, both theoretically and experimentally. Such spontaneous pattern formation requires the presence of a coupling between different points within the nonlinear medium. In optical systems this coupling is facilitated by the interplay between diffraction and nonlinearity. Experiments with nonlinear optical systems allow observation of the result of the interaction of many physical phenomena and reveal a rich variety of interesting effects, both stationary and dynamically evolving in time. One of the more recent nonlinear optical phenomena to be studied is optical parametric down-conversion (OPDC) in second-order $\left(\chi^{(2)}\right)$ nonlinear crystals in both the cases of a single pass [optical parametric amplifier, OPA] and with the crystal enclosed in an optical resonator [optical parametric oscillator, OPO]. The present paper is devoted to the experimental study of pattern formation in a transverse multimode OPO operating well above threshold. $\chi^{(2)}$ systems are interesting in their own right as examples of nonlinear interactions and generators of complex optical intensity patterns, and from a study of these phenomena we can learn much about the underlying physical effects and processes. However, these systems are also interesting from the point of view of practical applications. The OPA and OPO are important devices for the generation of coherent light with special properties. Moreover, these systems are examples of macroscopic quantum systems with properties that can only be understood and exploited with the help of quantum theory. The quantum properties manifest themselves as sub-Poissonian noise or quadrature noise squeezing and through quantum correlations between the intensity fluctuations of different optical modes in the transverse optical field (spatial quantum effects). The experimental requirements are demanding even for the measurement of classical multimode patterns: mechanically stable optical

\footnotetext{
*Electronic address: mlassen@fysik.dtu.dk
}

resonator, good thermal control, and high spatial and temporal image resolution. The measurement of quantum phenomena further requires high quantum efficiency and negligible thermal noise. Spatial quantum effects may open new applications in many different areas such as biophotonics, quantum information, and astronomy. Some actual examples are the measurement of small transverse displacements of a light beam below the standard quantum limit [1], the detection of weak phase images [2], quantum teleportation of optical images [3], and noiseless image amplification [4].

We present here our observations of multimode emission from a confocal OPO. In the near field we observe multiple beams and interference effects. In the far field complex structures are observed in the central lobe, which in cavities without nonlinearities is a smoothly varying, Gauss-like spot. These complex patterns are an indication that the OPO is operating in multiple modes with nonlinear coupling between the different fields. In the following we first provide an overview of the predicted experimental properties of multimode OPO's. Next we describe our experimental setup. We then present some of our experimental results followed by a discussion of their significance and their sensitivity to various experimental conditions. Finally, we conclude and present an outlook for further work.

\section{REVIEW OF EXPERIMENTAL PREDICTIONS AND RESULTS}

In this paper we consider the nonlinear process of parametric down-conversion of an incident pump wave, which through the nonlinear polarization of the medium, interacts with two other fields, the signal and idler fields-in particular, the case of an OPO above threshold. This downconversion process is governed by the simultaneous conditions of energy conservation and phase matching (momentum conservation). The process is also influenced by a number of effects that occur in the practical realization of such systems such as beam walk-off, thermal lensing, passive losses, and feedback effects. These effects complicate the comparison of experiment to theory. Experimental results 
are relatively few compared to the rich literature on theoretical results, so in our opinion there is a strong need for further work to study experimentally the various modes of operation of the OPO. In this section we first review the available experimental results and comment on to which degree they confirm the theoretical models.

Spontaneous OPDC pumped by a focused laser beam radiates into the surrounding free space, and the radiation pattern is basically a speckle pattern whose graininess is determined by the size of the pump region. The average speckle spot is a Fourier transformation of the near-field aperture. Thus the speckle spot size (coherence area) corresponds to the image resolution of the system (diffraction in the system pupil aperture). The underlying twin-photon generation is revealed by spatial and temporal correlations between diametrically opposing regions defined by the photon momentum conservation and the coherence condition. The problem can be treated theoretically in a linearized Langevin equation approach where the pump beam is considered a classical wave and the signal and idler beams consist of relatively small fluctuations with a zero mean [5]. The Langevin noise terms are related to vacuum quantum fluctuations. Theory predicts spatial squeezing and quantum correlations between the diametrically opposing coherent regions in the transverse plane-the so-called quantum images [6,7]. As the gain is increased by increasing the pump power, the fluctuations are amplified and the pulsations may contain a high number of photons. The time scales for these fluctuations are determined by the gain bandwidth of the optical system. Quantum experiments have been performed with single-photon detectors and with cooled charge-coupled-device (CCD) detectors and picosecond lasers [8-10]. In this region a high degree of agreement has been obtained between theory and experiment.

When the nonlinear medium is surrounded by an optical resonance cavity (OPO case), the down-converted signal and idler fields are radiated into the available cavity modes described by expansion in, for example, Gauss-Laguerre or Gauss-Hermite eigenmodes. To facilitate pattern generation a resonator with a high degeneracy for transverse modes is chosen. This could be a plane mirror cavity, which is often the starting point for a theoretical analysis, or a confocal or concentric cavity, which is more realistic for experimental work. The problem may be treated classically by expanding the field in, e.g., Gauss-Laguerre modes and computing the stability and resonating modes by solving the nonlinear propagation equations through the crystal.

Below threshold, the signal and idler fields are zero according to the semiclassical theory. However, in a full quantum description the signal and idler fields are zero only on the average, but contain a wide spectrum of temporal quantum fluctuations. Well below threshold the signal and idler fields are fluctuating both in the far field and in the near field and display basically a white-noise intensity distribution. Any perturbation of the homogeneous state is damped and decays. As one gets closer to the threshold, the fields acquire an increasing level of spatial order. In this regime the fluctuations show a precursor of the roll pattern that appears above threshold. This regime also belongs to the quantum imaging regime, where spatial (local) squeezing and spatial quantum correlations between small symmetrical regions (coherent regions) are found [7].

Conditions very near threshold can be described by the semiclassical theory where the pump field is regarded as a classical field [11]. Setting the time derivatives of the amplitudes equal to zero provides the steady-state solutions of the down-conversion process. Immediately above threshold a homogeneous solution is allowed for the generated signal and idler fields in a degenerate OPO, and a roll pattern appears in the signal and idler near fields due to interference between the signal and idler fields [12]. The pattern corresponds to two wave vectors whose transverse components are determined by momentum conservation and cavity detuning. This means that a slight cavity detuning allows an offaxis emission. In the far field, however, the two plane waves give rise to two localized spots. Experimentally, the roll pattern is difficult to observe unless some kind of phase locking is introduced between the idler and signal [13] as true degenerate operation is difficult to achieve in practice and leads to highly unstable interference patterns [14]. Higher-order nonlinear patterns have been observed, but mostly in systems that are nondegenerate in polarization so that signal and idler can be separated. In the work by Marte et al. [15] they employ the paraxial and mean-field approximation to obtain coupled equations for the transverse dynamics of the pump and signal fields for a degenerate $\mathrm{OPO}$ with spherical mirrors and finite-size input beam. The numerical solutions show various forms of patterns: rolls, spirals, pointlike structures, and others, which appear at various levels above threshold. The work by Schwob et al. [16] illustrates the richness of predicted patterns in a degenerate or nondegenerate triply resonant OPO: The device may operate in a single pair of signal and idler modes with the lowest threshold or it may operate with two or more thresholds, in a self-pulsed manner and in a phase-locked manner. One could perhaps say that in this region it would be desirable with more detailed investigations of the agreement between theory and experiment.

For pump powers well above threshold and for negative detuning, complex patterns may appear in the signal and idler far fields, observed by Vaupe et al. [17]. These complex patterns cannot be described as the interference between multiple signal and idler fields. The patterns arise from the coupling of many different modes due to consecutive nonlinear up-conversion and down-conversion processes. Well above threshold the signal and idler fields can recombine to form new pump beams that in turn generate new signal and idler fields with higher harmonics of the off-axis wave vector component. In both the signal and idler far fields, lattice like structures are observed in the central part of the beam (see the next section for some of our observations of these complex patterns). It is characteristic of these nonlinear patterns that they do not have the same structure in the near field and far field in contrast to mode patterns in a linear cavity, which do preserve the mode pattern throughout. As we shall see in the following, the patterns observed are different from those predicted by the theoretical models. The reason is probably that in the theoretical description of pattern formation approximations are often made, such as the plane-wave approximation and the thin-crystal approximation without absorption. Theoretical models become difficult to handle since 


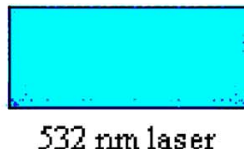

532 titn laser
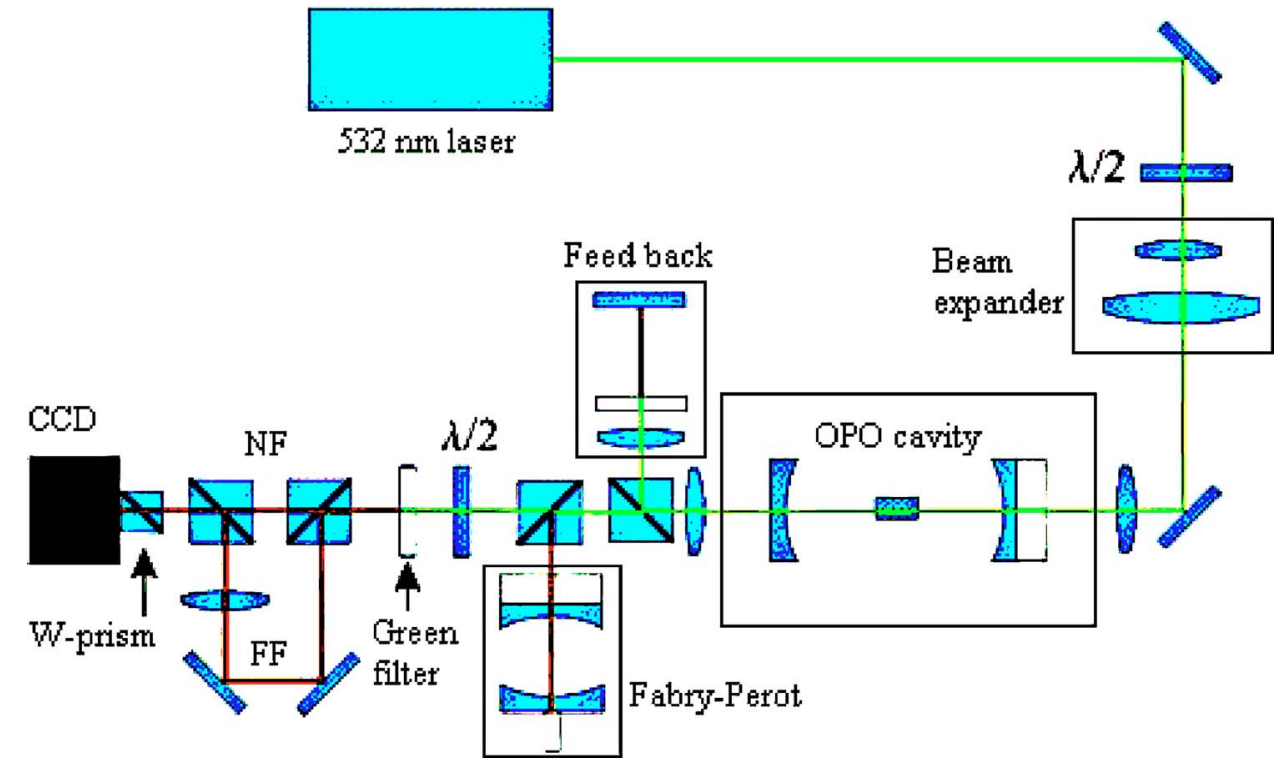

FIG. 1. (Color online) The experimental setup. linearization is no longer permissible. Instead numerical solutions containing a few (three to five) higher wave vector harmonics have been developed by Zambrini and San Miguel [18]. In high-gain situations even homogeneous solutions can develop pattern formation-the so-called spatial domains. Initiated by random fluctuations, multiple homogeneous solutions may develop simultaneously at different locations in the transverse plane. Because of the phase degeneracy, a phase difference of $\pi$ may exist between neighboring regions, and the homogeneous regions then display sharp dark bands where they meet, giving rise to typical domain patterns [19]. Such domain patterns have been observed in photorefractive oscillators, where the time scales are much more convenient for experimental observation [20], but so far not in parametric down-conversion, even though these devices are expected to behave in a similar fashion. The theory has been developed to include nondegenerate operation high above threshold [21]. In this case linearization is again permissible.

Theoretical models predict that the spatial quantum correlations, evident in subthreshold operation, survive in the high-gain region [18]; there is also some experimental evidence of this in the work by Martinelli et al. [22]. From an experimental and applications point of view, the high-gain region is very interesting because it provides a bright source of spatially quantum-correlated light. However, some practical concerns that are difficult to include in the models complicate comparison to experiment. First, non critical operation in a birefringent $\chi^{(2)}$ crystal is bound to introduce a directional preference. One consequence is that the different interacting fields propagate in different directions inside the crystal, the so-called beam walk-off. Thus walk-off breaks the rotational symmetry in the transverse plane, and the OPO, at the absolute instability threshold, selects traveling waves propagating in the walk-off direction among an infinity of unstable spatial and temporal modes [21,23]. Examples of this broken symmetry will be shown in the section about the observation of patterns. Walk-off has been shown to introduce a convective regime similar to convective instabili- ties in fluid flow. Theoretical models predict a lowering of the threshold for roll pattern generation initiated by noise (noise sustained patterns) [21]. However, competing regions may again develop well above threshold and form domains. Due to the convective terms introduced by the walk-off effect, these domains are predicted to drift in the walk-off direction while continuously regenerating due to new amplified-noise fluctuations. The situation resembles that of convective subharmonic domains experimentally observed and described by Buchhave et al. [24]. So far, no such convecting domains have been observed in OPO's, although a distortion of the average intensity pattern has been observed by Nishikawa and Uesugi [25]. Neither has anyone observed the predicted lowering of the threshold at the onset of oscillations due to amplified spontaneous noise. To further complicate matters and impede comparison with theory, experimental complications arise due to the unavoidable heating of the nonlinear crystal. The resulting thermal lens changes the cavity stability condition away from the transverse degeneracy (see later in this paper). Furthermore, the difference in refractive index for signal and idler in a type-II process means that signal and idler do not have the same confocal cavity length and do not excite the same mode patterns. Later in this paper we address this question of mode selection and show the influence of the polarization effects in the crystal.

If a well-defined mode pattern can be generated, the system will be more conducive to experimental investigation and to potential applications in multichannel quantuminformation systems. A few well-defined modes may also be more amenable to theoretical studies, although it is likely that the mode couplings will be highly nonlinear. As we shall show later, we have obtained experimental evidence that mode selection is possible by a feedback (self-seeding) procedure.

\section{EXPERIMENTAL SETUP}

The experimental setup is shown in Fig. 1. The figure shows the 532-nm pump laser, the pump beam mode match- 


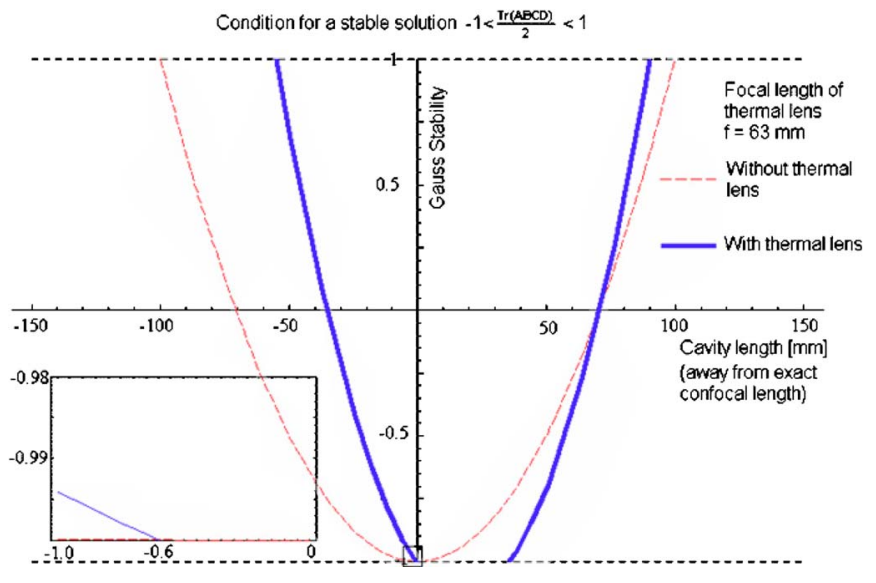

FIG. 2. (Color online) The Gauss stability as a function of cavity length change away from the exact confocal length. The thermal lens is $f=63 \mathrm{~mm}$ (pump power $1 \mathrm{~W}$ ). The dashed curve is without thermal lens and the solid curve is with thermal lens. In the small box the deviation between the unpumped (without lens) and pumped (with lens) can be seen.

ing optics, the OPO cavity, the feedback scheme, and the detection optics. The pump beam is a Gaussian TEM $_{00}$ beam from a 5-W Coherent VERDI CW frequency-doubled $\mathrm{Nd}$ :YAG laser. The OPO resonator is a confocal standingwave cavity resonant for the signal and idler beams. We have used a single-pass pump or a low-finesse pump resonance (pump-enhanced cavity). Previous experimental studies of patterns used a triply resonant cavity $[17,26]$, but this cavity is very unstable, and since we have no problems reaching the threshold for oscillation, we have chosen to work with the more stable doubly resonant cavity. The confocal cavity consists of two concave mirrors with radius of curvature $r$ $=-100 \mathrm{~mm}$ and high reflectance $(R>99.9 \%)$ for both signal and idler. Both mirrors are mounted on translation stages so that the coarse length of the OPO cavity can be varied by a few $\mathrm{mm}$ around the confocal length, $l_{\text {con }}=r+l_{\text {cryst }}(1-1 / \mathrm{n})$, where $l_{\text {cryst }}$ is the length and $n$ is the refractive index of the crystal. The mirror at the pump side is mounted on a piezoelectric element so that the cavity length can be scanned a few micrometers and adjusted to a resonance.

The pump beam waist is controlled by means of a variable-beam expander placed in the pump beam followed by a focusing mode-matching lens. This enables us to vary the pump beam waist from around $40 \mu \mathrm{m}$ up to about $300 \mu \mathrm{m}$. The beam waist of the confocal cavity $\mathrm{TEM}_{00}$ eigenmode is $92 \mu \mathrm{m}$ for the pump at $532 \mathrm{~nm}$ and $130 \mu \mathrm{m}$ for the signal and idler fields at $1064 \mathrm{~nm}$. In order to observe complex patterns the pump mode has to be wider than the $\mathrm{TEM}_{00}$ beam waist of the cavity [17]. However, when the pump beam waist is increased, more modes will be excited and the expected threshold will be higher. Assuming a pump beam waist of $250 \mu \mathrm{m}$ and the appropriate Boyd-Kleinmann factor [27], we find a theoretical single-mode threshold for this system of approximately $30 \mathrm{~mW}$. Pumping the confocal OPO with this pump beam waist and using negative detuning, we observed that the multimode threshold for the system was approximately $500 \mathrm{~mW}$. The threshold of the multimode OPO is roughly given by the single-mode threshold times the number of transverse modes [28]. Thus, based on the theoretical single-mode threshold and the observed multimode threshold we estimate that the OPO should be oscillating in a superposition of approximately 15-20 higher-order modes. The nonlinear crystal used is a $5 \times 3 \times 3 \mathrm{~mm}^{3}$ potassium titanyl phosphate (KTP) crystal cut in the $x y$ plane to allow type-II phase matching at room temperature. Both end surfaces are antireflection coated for pump, signal, and idler. The crystal is temperature controlled with a Peltier element. Since the refractive indices for signal and idler are different, the exact confocal lengths for these fields are different as can be seen from the equation for the confocal length above. Taking the average of the confocal lengths for signal and idler, we get the value $l_{\text {con }}=102.2 \mathrm{~mm}$, which we will use as our definition of the confocal length of the cavity. The difference in confocal length for signal and idler is small enough that both fields are multimode, but still significant enough that the mode patterns for the two fields will be different, as we shall see later.

Inside the crystal significant thermal effects take place due to absorption of the green light, which is approximately $3 \%$ at $532 \mathrm{~nm}$, and of infrared light, about $0.5 \%$ at $1064 \mathrm{~nm}$. The thermal index change causes the optical path length for a round trip to change. Thus a subconfocal cavity can become confocal if the pump power is sufficiently high. Using a pump power of $1 \mathrm{~W}$, we observe a confocal length of the OPO of $101.6 \mathrm{~mm}$, giving a deviation from the unpumped length of $-0.6 \mathrm{~mm}$. Using an $A B C D$ matrix method the exact confocal length with a thermal lens effect in the KTP crystal can be calculated. We find that a thermal lens of focal length $63 \mathrm{~mm}$ results in a confocal cavity length of $101.6 \mathrm{~mm}$, corresponding to the observed deviation from the unpumped confocal cavity length. This change of the confocal length due to thermal lensing is shown in Fig. 2.

The relation between the focal length of the thermal lens $f_{\text {ther }}$ and the pump power $P$ can be calculated from the variation in refractive index due to the heating of the crystal [29]. We find that for KTP the relation is given by $f_{\text {ther }}$ $=0.075(\mathrm{~m} \mathrm{~W}) / P$, giving a focal length of $75 \mathrm{~mm}$ when pumping the OPO with $1 \mathrm{~W}$. The reason why we are interested in the exact confocal length of the pumped cavity is that true multimode emission should occur for lengths slightly smaller than the exact confocal length. As shown previously by Vaupel et al. [17] and Ducci et al. [26] and as we shall describe later, the high-spatial-frequency interactions of the higher-order modes occur for cavity lengths just below the confocal length. Thus it is very important to know the exact confocal length as a function of pump power.

The green pump beam transmitted through the cavity is effectively removed by means of a low-pass optical filter. The image acquisition of the signal and idler is performed by means of an externally triggered, single-shot frame grabber. The CCD camera used is a JAI cv-m50IR extended infrared sensitivity with a relative low quantum efficiency at 1064 $\mathrm{nm}$. The exposure of images can be varied from $17 \mathrm{~ms}$ to $100 \mu$ s. The cavity was stable enough to allow up to 100 consecutive images to be recorded. Each pattern is averaged over the exposure time, which means that the observed fluctuations from image to image are limited to relatively low temporal frequencies. Observation of the whole range of dy- 


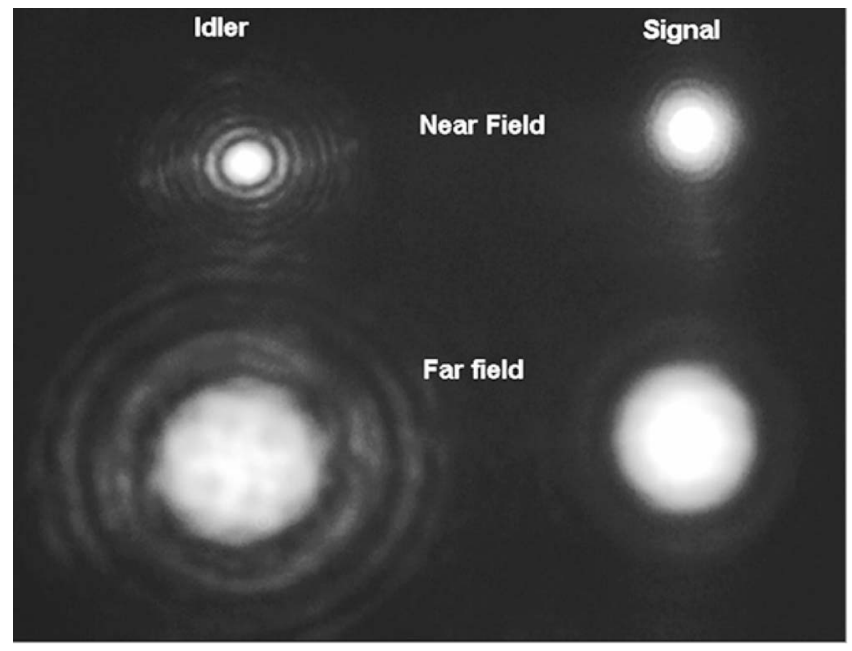

FIG. 3. Multimode fields in a confocal, doubly resonant OPO. Cavity length $L=101.5 \mathrm{~mm}$.

namic fluctuations would require much faster sampling times, which we consider obtaining by double-pulsed $Q$-switched operation of the pump.

The output beam from the OPO is split into two separate paths and then recombined. A suitable choice of lenses in the two branches allows both near field and far field to be imaged simultaneously on the CCD camera. Since KTP is a type-II nonlinear crystal, the signal and idler can further be separated by a polarizing beam splitter. A Wollaston prism polarizer is placed just in front of the CCD detector to introduce an angle difference of $10^{\circ}$ between the two polarizations. Thus we obtain simultaneous imaging of signal and idler near field and far field by splitting the output from the OPO into four beams and separately adjusting the imaging of each. Figure 3 shows the position of the four fields as they appear in the CCD image. We shall use these locations of the four beams throughout the paper.

\section{OBSERVATION OF PATTERNS}

The primary goal of the experimental work is the study of the confocal cavity OPO far above threshold. Figure 3 shows a typical simultaneous recording of the signal and idler near field and far field of the OPO with a pump power well above threshold and for negative detuning. We observe that both the near field and far field include a number of concentric rings. The structure of the central part is not visible due to saturation of the detector. To see this part the intensity must be reduced, which then prevents observation of the outer rings. The concentric rings were only observed for cavity lengths smaller than the confocal length, which indicates that the rings are not just due to diffraction in the crystal, but are higher-order cavity modes. Here 10-15 rings can be counted in the idler near field, which is in good agreement with the threshold analysis in the previous section. Figure 4(a) shows a superposition of the first 10 even Gauss-Laguerre modes $\left(\mathrm{TEM}_{0,0}\right.$ to $\left.\mathrm{TEM}_{18,0}\right)$. From this simulation we obtain an intensity distribution in good agreement with the measured idler near-field pattern in Fig. 4(b). We are thus confirmed in
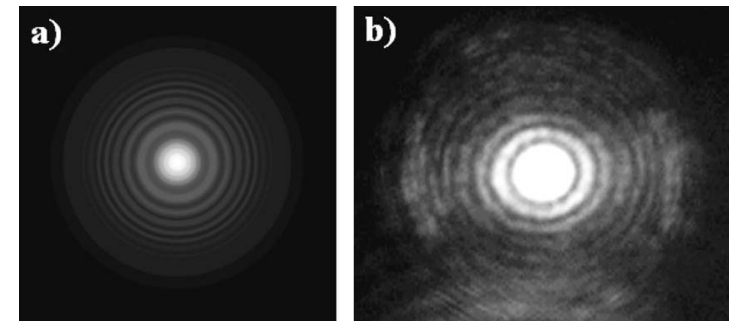

FIG. 4. (a) Simulation of a superposition of ten Gauss-Laguerre modes. (b) Idler NF consists of a central spot surrounded by concentric rings (Gauss-Laguerre-like modes). Cavity length $L=101.5 \mathrm{~mm}$.

our conclusion that the OPO oscillates on a coherent superposition of roughly $10-15$ transverse modes.

Figure 5 shows a scan with a Fabry-Perot spectrum analyzer with 8-GHz FSR. From the scan can it be seen that the OPO oscillates in one longitudinal mode. Thus the observed transverse modes belong to one degenerate longitudinal mode as expected. This observation is in good agreement with the description of the triply resonant OPO in the work by Vaupel et al. [17].

In Figs. 6 and 7 we see that the transverse structures of the near field and far field are different for both signal and idler. This indicates that the structure is due to the nonlinear interaction between many modes in the crystal. This leads to the following definition of multimode emission from a nonlinear source: When the near-field and far-field structures of the intensity pattern are different the nonlinear source emits multimode light $[22,26]$. Figures 7 (a) and 7(b) show the central part of the idler near field and far field for a cavity length of $101.5 \mathrm{~mm}$. It can be seen that the near field and far field have different transverse patterns, indicating a multimode nonlinear interaction. We further observe that the structure of the signal and idler pattern is different, both in the near field and in the far field. This is a result of the birefringence of the crystal, which causes the detuning from the exact confocal length to be different for signal and idler and also introduces an asymmetry due to beam walk-off.

To test the effect of the difference in cavity optical length for the signal and idler we changed the cavity to a semiconfocal one with a plane mirror placed in the center and the crystal placed as close to the plane mirror as possible. In this type of cavity the walk-off effect is compensated outside the crystal and also as seen from outside the cavity (but not inside the crystal itself). Tuning the length of the cavity slightly around the confocal length we observe that the signal cavity mode changes into a mode that looks very much like the idler cavity mode before the tuning of the length and vice versa (see Fig. 8). The difference between the signal and idler cavity modes is due to non-negligible walk-off inside the crystal. From this observation we conclude that the difference in refractive index for the signal and idler due to the type-II crystal causes the signal and idler to have different resonance lengths for the different modes.

In the semiconfocal cavity we were also able to observe high-order Gauss-Ince modes. These modes have only recently been observed for the first time in laser fields [30], and this is the first time that such patterns have been ob- 


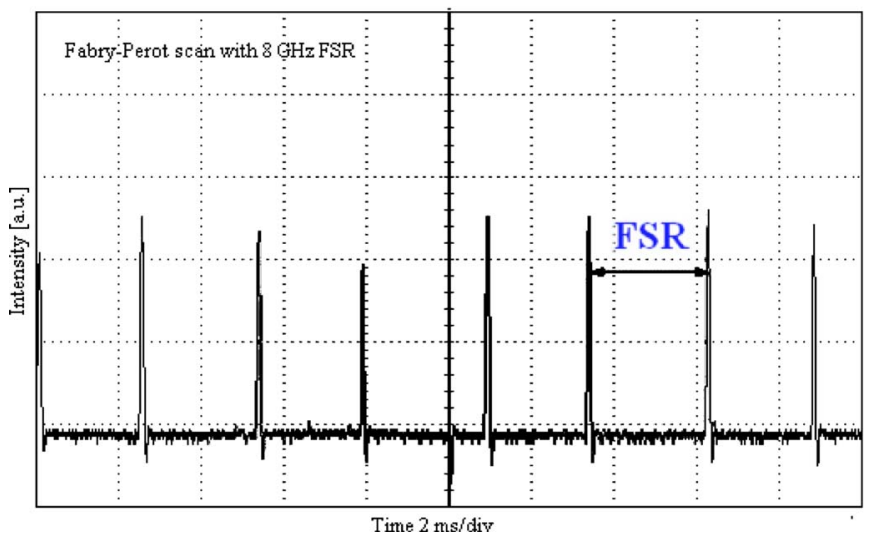

FIG. 5. (Color online) Fabry-Perot scan of OPO output showing the longitudinal modes.

served in an OPO output. In Fig. 8 the patterns look like a superposition of a single high-order Gauss-Ince mode and the Gaussian fundamental mode. The Gauss-Ince modes form a complete family of orthogonal solutions to the paraxial wave equation just like the Gauss-Laguerre modes and the Gauss-Hermite modes, but the high-order Gauss-Ince modes are favored when a transverse asymmetry is present in the cavity.

In the central part of the signal and idler far field we observed complex, latticelike patterns. These complex patterns contain details, which are much smaller than the Gaussian $\mathrm{TEM}_{00}$ mode, which represents the smallest spatial mode pattern of the linear cavity. The appearance of patterns smaller than the $\mathrm{TEM}_{00}$ mode indicates the presence of cascaded nonlinear interactions in the crystal causing the formation of fields with higher harmonics of the transverse wave vector. The appearance of the patterns changes when the cavity length is changed. We have observed that the spatial frequencies of the complex patterns increase when the cavity length is made shorter; see Fig. 9. Our observation of complex latticelike patterns in the signal and idler fields is in good agreement with the observations made by Vaupel et al. [17].

From the figures above we are led to the conclusion that the observed degenerate type-II confocal OPO for a cavity

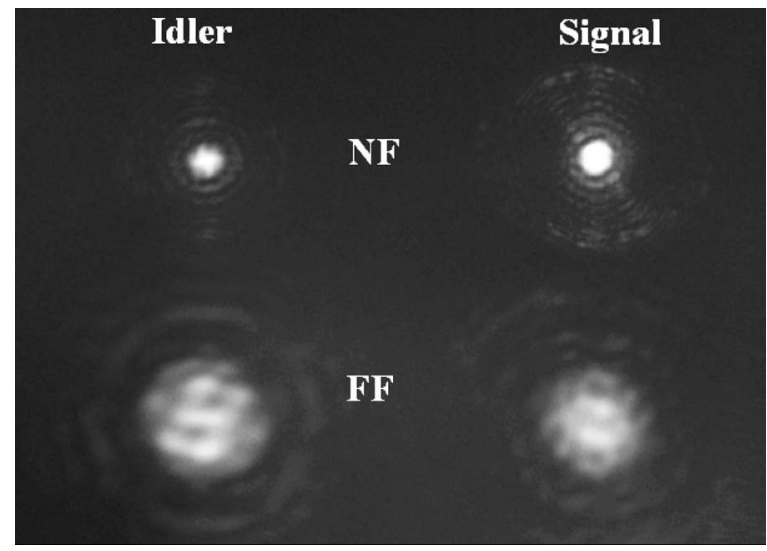

FIG. 6. Different transverse structures of the signal and idler. Cavity length $L=101.1 \mathrm{~mm}$. length of $101.5 \mathrm{~mm}$ is operating as a multimode OPO with a nonlinear coupling between the modes. From the figures and threshold analysis we conclude that the OPO is oscillating in a superposition of approximately 15 eigenmodes. Since the primary condition for the occurrence of spatial quantum effects from an OPO is that many higher-order transverse modes be confined in the cavity, we conclude that this device is suitable for the further study of transverse quantum effects such as spatial squeezing and quantum correlations. However, our study also underlines the experimental difficulties that complicate the comparison to theoretical predictions. These difficulties are primarily associated with the properties of the crystal. The birefringence of the crystal, which allows the separation of signal and idler fields by polarization splitters, also causes the detuning from the confocal cavity length to be different for signal and idler, which in turn results in significantly different intensity patterns for the two fields. Due to the highly effective nonlinear coefficient, the crystal can be relatively short, which means that the walk-off effect does not significantly reduce the nonlinear conversion efficiency, but the walk-off does cause a breaking of the rotational symmetry inside the crystal, further complicating the experimental situation. Finally, the KTP crystal seems to have a too high absorption cross section for the green pump light, causing rather severe thermal effects. The thermal change of refractive index causes a change in optical cavity length with pump power, thus changing the detuning from the confocal cavity length. Furthermore, the thermal lens effect causes a change in stability of the cavity, further influencing the structure of the generated pattern. Finally, the deviation of the thermal lens from a perfect thin lens may cause diffraction. Although we have ascertained that the observed patterns are not primarily due to diffraction in the crystal, this diffraction may still cause unwanted cavity losses, reducing the conversion efficiency and any quantum effects. In future work we will try to find a crystal without walk-off, but still type II; this could possibly be a periodically poled KTP oriented for the type-II instead of the usual type-I PPKTK configuration. Another possible solution is to simply work at a shorter wavelength with a greater selection of crystals and higher quantum efficiency for the detector.

As expected, the multimode confocal OPO is rather unstable, and it is difficult to maintain a given mode pattern for more than a few seconds. For this reason and because it would be of great interest in quantum communications ex-
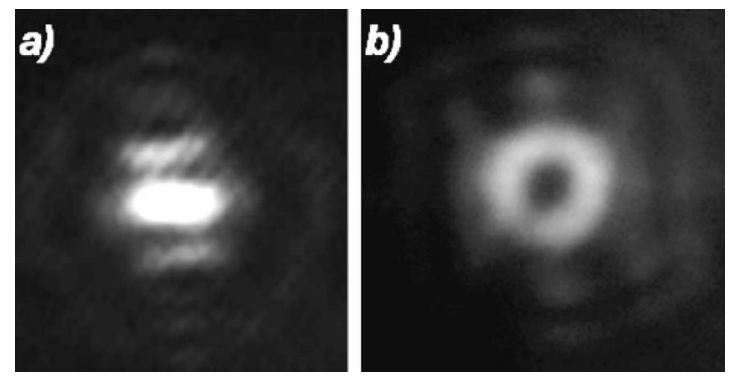

FIG. 7. (a) Central part of the idler near field and (b) central part of the idler far field. The near field and far field display different transverse patterns, which is an indication of multimode emission. Cavity length $L=101.5 \mathrm{~mm}$. 

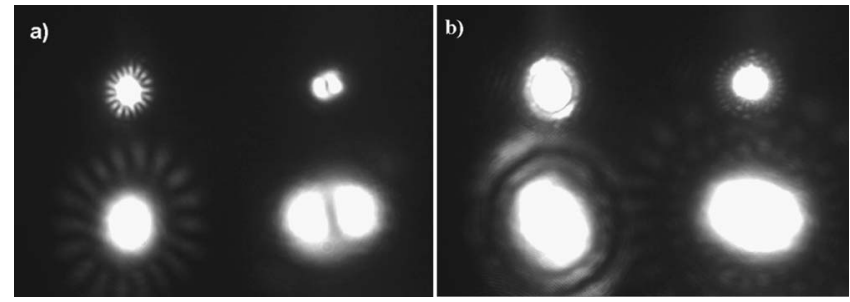

FIG. 8. Changing the fine-tuning of the cavity slightly around the exact resonance length. (a) The idler looks like a Gauss-Ince mode. (b) For larger negative detuning the signal changes into a pattern that looks like a Gauss-Ince mode.

periments to be able to design and stabilize a selected multimode pattern, we have made preliminary experiments to see if the confocal multimode OPO is sensitive to feedback and if the feedback seems to have a stabilizing effect on the mode pattern. The idea of the feedback is to make a phase modulation of the signal and/or idler and then seed one or both back into the OPO cavity and thereby excite some selected superposition of eigenmodes in the cavity. Only coupling between even modes or between odd modes is possible in this feedback procedure, since the transverse mode number must increment by 2 for the confocal cavity to operate on a given longitudinal mode. The feedback arrangement is show in Fig. 1. Part of the signal and idler beam was split off with a beam splitter with high transmittance for the pump and some reflectance for the signal and idler, and sent to a plane mirror through a mode matching lens. The plane mirror was HR coated for $1064 \mathrm{~nm}$. The plane mirror and the mode matching lens were mounted on a translation stage, which allowed fine-tuning of the feedback phase and the mode matching.

The conclusion of this preliminary test is that the cavity is sensitive to even small amount of feedback and that the feedback has a stabilizing influence on the mode pattern. Figure 10 shows an example of the emitted pattern with and without the feedback. Without feedback [Fig. 10(a)] we observe a $\mathrm{TEM}_{00}$-like pattern without structure. With feedback [Fig. 10(b)] a pattern forms and stays stable for a relatively long time. In future experiments we will insert a spatial phase modulator in the feedback path to try to control the pattern, a method which has previously proven successful in, e.g., solid-state lasers and photorefractive oscillators.

These first experiments have shown that the OPO is very sensitive to feedback and that higher-order modes probably can be excited and controlled by this procedure. At the present time we have developed a method for the detection of quantum correlations, and in order to succeed with this method we need active control of the higher-order modes. The detection method and our experimental data will be described in a forthcoming paper.

\section{CONCLUSIONS}

We have investigated the confocal, doubly resonant, pump-enhanced, multimode OPO above threshold. We have shown that this device is able to oscillate in a superposition of at least 15 transverse modes and can be stabilized to provide multiple recordings of the same mode pattern. Furthermore, we have observed high-spatial-resolution patterns inside the central lobe mode in the far field that must be ascribed to cascaded nonlinear interactions in the crystal. We have shown how the spatial scales of these far-field patterns evolve as a function of the length detuning from the confocal condition. The spatial frequency of these patterns increases as we approach the confocal cavity length from the inside until they suddenly disappear after passing to the positive side of the confocal cavity length.

As the confocal resonator is rather unstable for the multimode signal and idler fields (whereas the low-finesse pump resonance used by previous investigators in triply resonant OPO's is rather stable, which, however, does not guarantee that the cavity is stable for the signal and idler fields), we have made simultaneous four fold high-speed recordings of signal and idler in both the near field and far field. With this method we have succeeded in recording about 100 consecutive images of a given mode pattern to be used in planned measurements of temporal and spatial fluctuations of the intensity in order to investigate temporal squeezing and spatial
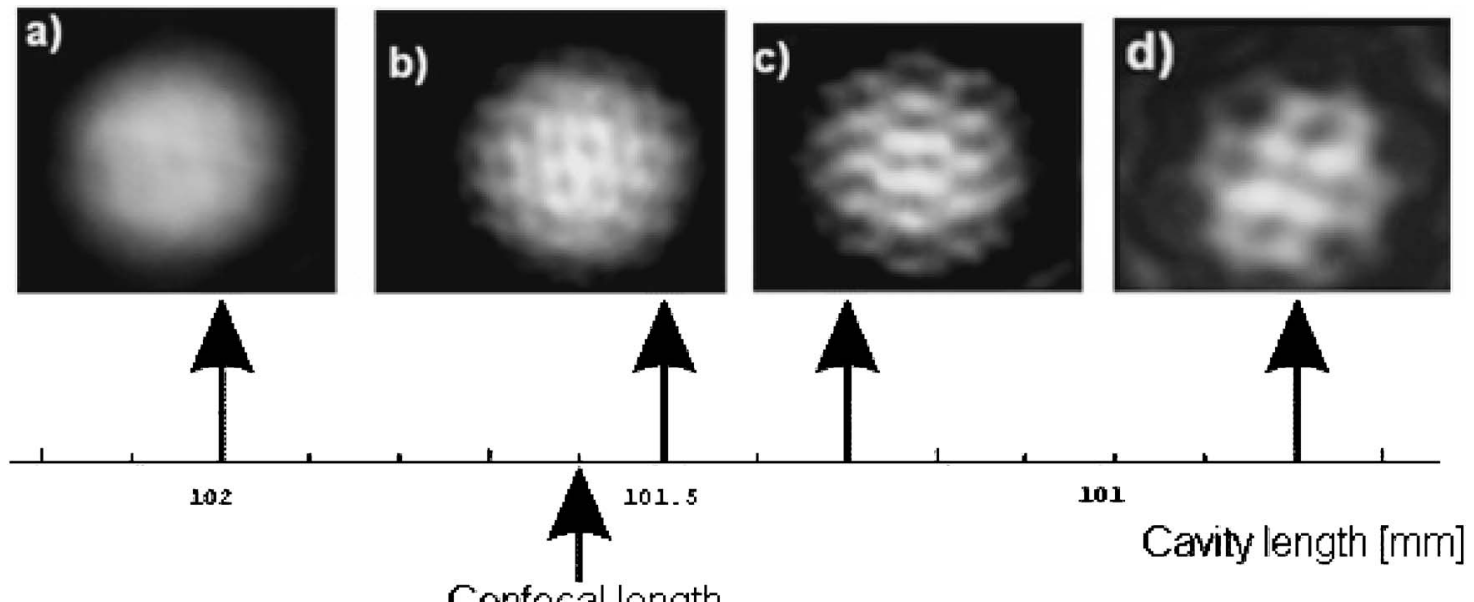

Confocal length

FIG. 9. The evolution of the complex signal far-field patterns as a function of the measured cavity length. (a) $L=102.0 \mathrm{~mm}$. (b) $L=101.5 \mathrm{~mm}$. (c) $L=101.3 \mathrm{~mm}$. (d) $L=100.8 \mathrm{~mm}$. All images are recorded with a gray filter. Observed confocal length $101.6 \mathrm{~mm}$. 

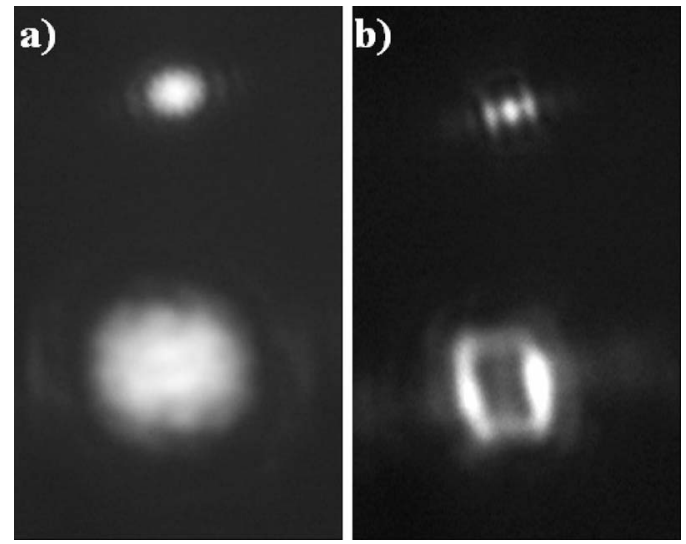

FIG. 10. Feedback experiment where the idler is forced into a pattern by feedback. (a) Without feedback. (b) With feedback.

correlations in the transverse far-field pattern.

Furthermore, we have investigated the effect of various experimental complications on the generated patterns. We have shown that thermal effects change the detuning from the confocal condition and also the stability parameter for the cavity, and we have shown that this effect can be compensated for by adjusting the cavity length in accordance with the pump power used. We have shown that the higher-order modes observed in the far field are due to the multimode character of the cavity and not a result of diffraction in the thermal index distortions caused by the heating of the crystal. Also, we have shown that the difference of the signal and idler patterns is due to the birefringence of the crystal and, by investigating the related semiconfocal cavity, we have shown that it is not possible to compensate for this effect because it originates internally in the crystal. We have shown that it is possible to change the idler cavity mode to the signal cavity mode and vice versa by small adjustments of the cavity length, which shows the consistency of the mode pattern formation. In the same experiment we observed, in an OPO, higher-order so-called Gauss-Ince modes. These modes are eigenmodes in an alternative expansion of the field in a slightly asymmetric paraxial cavity. Finally, we have determined by preliminary experiments that the intensity patterns for the signal and idler fields of the multimode confocal OPO above threshold are very sensitive to feedback and that even a primitive feedback of all the modes by a plane mirror stabilizes the pattern to some degree. We thus predict that it will be feasible to design and control the mode pattern by a feedback scheme, which utilizes a spatial phase modulator in the feedback arm.

In summary, we have shown that the main condition for the occurrence of spatial quantum effects from an $\mathrm{OPO}-$ namely, the occurrence of many higher-order transverse Gaussian modes in the OPO cavity-is fulfilled in the doubly resonant, confocal OPO above threshold. The fact that we can now obtain the simultaneous near field and far field of both signal and idler beams on the same CCD frame ensures the validity of correlations between these fields. This is an important feature in future experiments, where we plan to make direct detection of quantum correlations between the signal and idler far fields.

\section{ACKNOWLEDGMENT}

This work was supported by Danish Technical Research Council (STVF) Project No. 26-03-0304.
[1] N. Treps, U. Andersen, B. Buchler, P. K. Lam, A. Maitre, H. A. Bachor, and C. Fabre, Phys. Rev. Lett. 88, 203601 (2002).

[2] L. A. Lugiato, A. Gatti, and E. Brambilla, J. Opt. B: Quantum Semiclassical Opt. 4, 176 (2002).

[3] I. Sokolov, M. Kolobov, A. Gatti, and L. A. Lugiato, Opt. Commun. 193, 175 (2001)

[4] M. Kolobov and L. A. Lugiato, Phys. Rev. A 52, 4230 (1995).

[5] A. Gatti, H. Wiedemann, L. A. Lugiato, and I. Marzoli, Phys. Rev. A 56, 877 (1997).

[6] L. A. Lugiato, A. Gatti, and H. Ritsch, J. Mod. Opt. 44, 1899 (1997).

[7] M. I. Kolobov, Rev. Mod. Phys. 71, 1539 (1999).

[8] O. Jedrkiewicz, Y.-K Jiang, E. Brambilla, A. Gatti, M. Bache, L. A. Lugiato, and P. Di Trapani, e-print quant-ph/0407211, v1.

[9] F. Devaux and E. Lantz, Eur. Phys. J. D 8, 117 (2000).

[10] M. L. Marable, S. K. Choi, and P. Kumar, Opt. Express 2, 84 (1998).

[11] A. Gatti, L. A. Lugiato, G-L. Oppo, R. Martin, P. Di Trapani, and A. Berzanskis, Opt. Express 1, 1 (1997).

[12] G-L. Oppo, M. Brambilla, and L. A. Lugiato, Phys. Rev. A 49, 2028 (1994).

[13] L. Longchambon, J. Laurat, T. Coudreau, and C. Fabre, e-print quant-ph/0311123, v2.
[14] M. Le Berre, E. Ressayre, and A. Tallet, Phys. Rev. E 67, 066207 (2003).

[15] M. Marte, H. Ritsch, K. I. Petsas, A. Gatti, L. A. Lugiato, C. Fabre, and D. Leduc, Opt. Express 3, 71 (1998).

[16] C. Schwob, P. F. Cohadon, C. Fabre, M. A. M. Marte, H. Ritsch, A. Gatti, and L. Lugiato, Appl. Phys. B: Lasers Opt. 66, 685 (1998).

[17] M. Vaupel, A. Maitre, and C. Fabre, Phys. Rev. Lett. 83, 5278 (1999).

[18] R. Zambrini and M. San Miguel, Phys. Rev. A 66, 023807 (2002).

[19] K. Staliunas and V. J. Sanchez-Morcillo, Phys. Rev. A 57, 1454 (1998).

[20] V. B. Tarananko, K. Staliunas, and C. O. Weiss, Phys. Rev. Lett. 81, 2236 (1998).

[21] H. Ward, M. N. Ouarzazi, M. Taki, and P. Glorieux, Eur. Phys. J. D 3, 275 (1998).

[22] M. Martinelli, N. Treps, S. Ducci, S. Gigan, A. Maitre, and C. Fabre, Phys. Rev. A 67, 023808 (2003).

[23] H. Ward, M. N. Ouarzazi, M. Taki, and P. Glorieux, Phys. Rev. E 63, 016604 (2001).

[24] P. Buchhave, S. Lyuksyotov, M. Vasnetsov, and C. Heyde, J. Opt. Soc. Am. B 13, 2595 (1996). 
[25] T. Nishikawa and N. Uesugi, Opt. Commun. 124, 512 (1996).

[26] S. Ducci, N. Treps, A. Maitre, and C. Fabre, Phys. Rev. A 64, 023803 (2001).

[27] G. D. Boyd and D. A. Kleinman, J. Appl. Phys. 39, 3597 (1968).
[28] S. Gigan (unpublished).

[29] W. Koechner, Solid-State Laser Engineering (Springer-Verlag, Berlin, 1996).

[30] U. T. Schwarz, M. A. Bandres, and J. C. Gutiérrez-Vega, Opt. Lett. 29, 1870 (2004). 